\title{
Apple slide tachistoscope
}

\author{
TERRENCE C. BIGGS and DANIEL PULHAM \\ University of Western Ontario, London, Ontario, Canada \\ and \\ FRASER A. BLEASDALE \\ Trent University, Peterborough, Ontario, Canada
}

\begin{abstract}
We outline a BASIC program and subsidiary machine language timing routines that enable the Apple II series of computers to be employed as control devices for the tachistoscopic presentation of 35-mm slides and the collection of response latencies. The program is functional for experiments that require pictorial stimuli to be presented in up to three separate fields, with precise display times, precise interstimulus and intertrial intervals, and the collection of manual or vocal reaction times with millisecond accuracy. The major advantages of this system are that initial setup costs are substantially lower than those for other similar systems (e.g., Loftus, Gillispie, Tigre, \& Nelson, 1984) and that user customization of the program may be accomplished by anyone possessing rudimentary knowledge of the BASIC programming language.
\end{abstract}

Although the Apple II series of computers are equipped with four usable graphics screen pages, their use for tachistoscopic (T-scope) presentations is limited. The most obvious drawback is that two of the four pages are constrained to the presentation of text material or lowresolution graphics. Thus, three-field $\mathrm{T}$-scope presentations requiring equivalent-resolution graphics (other than text materials) are possible only if stimuli presented on the high-resolution graphics pages are degraded to the level of low-resolution graphics presentations or if three high-resolution stimuli can be loaded and presented rapidly. Rapid presentation of stimuli is unlikely to pose a problem if a relatively limited set of items is employed and/or if the items consist of simple geometric shapes; however, use of the video monitor for stimulus presentation may be hampered by memory limitations if many different stimuli are required (Fraser, Lishman, \& Parker, 1987).

Constraints on the number of stimuli that may be preloaded into RAM may make it necessary to access the disk for additional stimuli. Accessing the disk for stimuli is usually unsatisfactory, however, because it requires considerable time. For example, BLOADing one of Prill's (1984) picture stimuli and toggling on the appropriate screen requires an average of $4,250 \mathrm{msec}$. An interstimulus interval (ISI) of this magnitude is far too long if a procedure requires rapid sequential pic-

The development of this system was supported in part by Grant $\mathrm{A6333}$ from the Natural Sciences and Engineering Research Council of Canada to Stephen J. Lupker. We thank R. St. John, J. Scheuhammer, and R. Wright for comments and suggestions on earlier drafts of this paper. Special thanks to A. Patrick for assistance in improving the accuracy of the software timing routines. Address correspondence to Terrence C. Biggs, Department of Psychology, University of Western Ontario, London, ON N6A 5C2, Canada. ture presentations. Conversely, if the goal of an experiment is to study performance on stimulus integration or fragmentation, and relatively few stimuli need to be shown in rapid succession, the use of the video monitor allows very accurate (i.e., pixel $\times$ pixel) spatial orientation of components, in comparison with manual orientation of component images projected on a viewing screen, and would be preferable to the system outlined in this paper. Finally, the resolution of the Apple II graphics may not be sufficient for the presentation of complex visuospatial stimuli (e.g., human faces). Indeed, investigations of stimulus fragments (Brooks, 1987; Snodgrass, Smith, Feenan, \& Corwin, 1987) and facial perception (Jensen, 1987) that have presented stimuli via a video monitor have involved the use of the more powerful Apple Macintosh computer with 512K RAM and $246 \times 246$ pixel resolution, which is better than the $280 \times 192$ pixel resolution available on the 48K RAM Apple II or II + and the $128 \mathrm{~K}$ RAM IIe.

The best solution to the problems of memory limitations and the concomitent effects on rapidity of stimulus presentation and of the resolution inadequacies with the Apple II series of computers is to employ the computer as a control device for an external set of apparatus. The computer may be interfaced directly with a T-scope that is equipped with an automatic card-delivery system; however, these mechanisms are subject to failure because the cards have a tendency to jam. This is particularly the case when the cards become worn, slightly warped, or damaged.

The most reliable external apparatus we have used consists of a set of Kodak carousel slide projectors coupled with electronic shutters, all of which are controlled by computer. A detailed description of the advantages and limitations of using 35-mm slides in a computer-based 
laboratory has been presented by Loftus (1982). Loftus, Gillispie, Tigre, and Nelson (1984) described a fairly sophisticated slide-based projector laboratory, but many of their presentation options may not be necessary for specific applications. The cost of their system in 1984 was also significant $(\$ 15,044$ U.S.). The Apple slide tachistoscope (AST) program presented here is somewhat less sophisticated (i.e., stimulus luminance is not under operator control, and responses may be obtained only from single subjects), but set-up costs (see Appendix) are substantially lower.

\section{Program Outline}

The AST program provides a means of controlling required external devices and reading input from contact or voice-operated keys. An outline of program control is presented in Figure 1. The program is written in Applesoft BASIC, and control of projectors and shutters is handled by a series of POKE commands channeled through a custom hardware card. The collection of response times and response categories is handled within a machine language timer routine. This routine is based upon the approach to timing detailed by Dorfman (1987, method 4) and provides a countdown timer (Dorfman, 1987, method 2) for use in setting ISI and intertrial interval (ITI) parameters. These routines are located in page 3 of memory, an area protected for monitor vector locations. The routines begin at HEX 0300 (decimal 768) and extend to HEX 0388 (decimal 904). This location is ideal, because the routines are safe from accidental overwriting by both programming software and data storage while presenting no interference with special monitor functions that start with autostart ROM at HEX 03F0 (decimal 1008) and continue to HEX 03FF (decimal 1023). Although the routines may be moved to any location in memory to suit the user, such a shift would necessitate complete rewriting of the assembly code to reflect the change in location, and changes to the decimal locations in the BASIC AST program's PEEK, POKE, and CALL addresses would be needed. Consequently, unless the timing routines are co-opted for use in other programs that require their current location for other functions, relocation of the routines is both unnecessary and undesirable.

A subject's responses are picked up via the game port through PEEKing a location that stores signals corresponding to the three on/off pushbutton switches, PB0, PB1, and PB2 (HEX C061, C062, and C063). Response times are obtained via a CALL to a routine that returns values to the main program, which, following calibration, are accurate within -1.3 to +.5 msec for times ranging between 0 and $3,300 \mathrm{msec}$. Corrections for this error are handled by the main program.

ISIs are set by POKEing an integer value in the range 1-255 into a storage area. The countdown is initiated with a CALL to a routine that loops until the storage location value is met. For example, POKEing the storage location with the value 7 and CALLing the countdown routine executes a 70 -msec delay interval. ISIs may be incremented in steps of 9 or $10 \mathrm{msec}$. It is important to note, however, that ISIs and ITIs may not be operated while the response timer is engaged. The accuracy of these timing routines is based upon a comparison of the values returned by the software and the values returned by the California Computer Systems (CCS) hardware clock-card.

Customization of the program is facilitated by the fact that it is written in Applesoft BASIC and all adjustments to the machine language timer and countdown routines may be made by using simple POKE commands. For example, the current implementation of the AST program controls three fields set up as prime, target, and mask and requires subjects to make a response to stimuli presented in two of the fields. To eliminate the required response for one of these fields, simply deleting a few lines of code that CALL the machine language timer and replacing them with a POKE to the storage location and a CALL to the countdown routine allows the field to be shown for a specified interval without requiring a response to terminate field presentation. Determination of stimulus presentation duration via the countdown timer allows a minimum timed exposure of $12 \mathrm{msec}$ coupled with a maximum of $2 \mathrm{msec}$ rise/fall time required by the commercial Gerbrands G1166 shutter. The ability to increment exposure duration in 9- or 10 -msec intervals represents a minimum improvement factor of 1.6 over the 16.67 -msec interval minimum imposed for presentations on the video monitor.

Control of the projectors is handled via POKE commands, and, in the present version of AST, the projectors presenting stimuli to fields one and two are advanced simultaneously. To obtain individual projector incrementation, an additional transistor and relay are required on the hardware card and another POKE value to the next sequential card address is necessary. Control over the shutters for the three fields is also handled by POKEing locations on the card. The other parameters of the program (i.e., number of trials, number of trial blocks) are assigned to user-determinable variables. The total number of trials is unlimited; however, the number of trials that may be presented in a single block is constrained by the number of slides that can be placed in a single carousel. Given a capacity of 140 slides per carousel, the current implementation of AST would be limited to 140 prime-target trials per block. Data is transfered to disk at the end of each block of trials. This manner of recording responses is particularly useful in experiments requiring large numbers of trials because, after any interruption of the program, the experimenter can resume data collection at the beginning of the trial block during which the interruption occurred rather than losing a subject's set of data. 


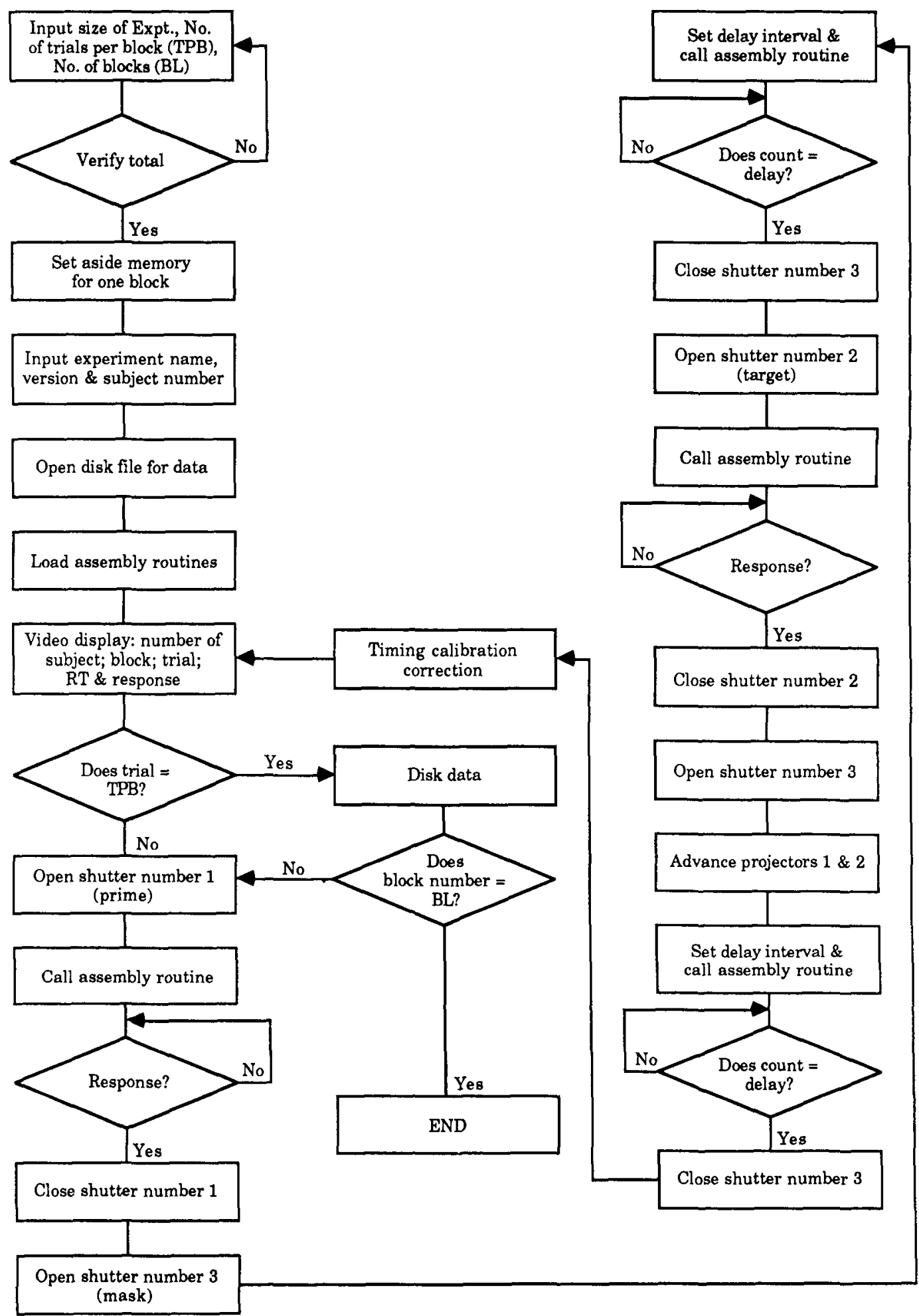

Figure 1. Flowchart of program control for the Apple computer slide tachistoscope. 


\section{Hardware Requirements}

The card that serves as the interface between the computer and the outside apparatus must reside in slot 5 for the current version of AST; otherwise, all of the POKE commands that operate the shutters and projectors must be changed to adjust for the new slot location. The card is easily assembled using a standard Apple-compatible 4609 Vector prototype board and requires the following components: four Potter and Brumfield T82 P11 D11105 dip relays, four $2 \mathrm{~N} 3439 \mathrm{NPN}$ transistors, three 74LS30 eight-input NAND gates, three 74LS373 octal transparent latches, two 74LSO4 hex inverts, one 74LSO2 quad two-input NOR gate, as well as cable and assorted connectors.

\section{General Control}

The discussion up to this point has stressed that the AST program can be used for the control of slide projectors; however, it is possible to use the program to control a wide variety of external devices. The limitation on external control in the present system is imposed by the strength of the dip relays. Use of the Potter and Brumfield T82 P11 D111-05 limits triggering of external devices to those that require up to a maximum of $2 \mathrm{~A}$ triggering current. To control devices requiring greater amperage, it is necessary to use the specified dip relays to trigger other relays capable of generating the required amperage.

The timing routines are BLOADed by the BASIC program and thus are available for use in other programming applications; however, the structure of the routines implements a technique that requires modifications to the firmware of the computer. The timer onset is synchronized to the raster pulse. This is necessary for accurate timing of presentations on the video monitor and requires a simple one-wire hardware modification of the motherboard for the Apple II and II+ computers (Lancaster, 1984). In the Ile and IIc computers, the location to check for the raster pulse is different and requires a POKE to the timing routine to change from HEX C063 to C019 (Dorfman, 1987). If you do not have this synchronization modification, then a single POKE disables the routine that checks for the pulse. For the purposes of AST, the raster check is not a necessary function, but if this timing routine is used in conjunction with screen presentations, without the modification or with the check disabled, the reaction times returned become subject to additional variance $(-8$ to $+8 \mathrm{msec})$. Consequently, use of this timer for screen presentations is not recommended without the modification to the motherboard of the Apple II and IIt computers.

The current version of AST advances fields one and two simultaneously and field three remains fixed. To extend control over each projector individually, it would be necessary to add one additional transistor and dip relay to the hardware card for each increment in control. The physical size of the 4609 Vector prototype board limits the addition of extra relays to a total of 16 , which limits external control to 16 devices (e.g., 8 projectors and 8 shutters). Assembling the components on a larger card would allow room for more relays, up to a maximum of 20 , prior to modification of prerelay circuit logic. This enlargement of the system would allow control of an additional two projectors and shutters, for a total of $20 \mathrm{ex}-$ ternal devices that require no more than a 2-A signal. The expansion of the hardware card can be accomplished at relatively little additional cost and involves only a simple extrapolation of the existing schematics. One major advantage of enlarging the system is tied to the delay that is imposed when advancement of a projector is required: The current version of AST presents three fields consecutively prior to advancing projectors, which allows for a maximum of three stimuli to be shown in $42 \mathrm{msec}$ and then imposes an interval of $800 \mathrm{msec}$ for projector advancement prior to the availability of the next stimulus for presentation. With such a lengthy interval required for projector advancement, experiments that require more than three consecutive presentations with ISIs of less than $800 \mathrm{msec}$ require modifications to allow additional projectors and shutters to be used. Extrapolation to the maximum possible on the specified 4609 card allows eight consecutive stimuli to be shown within $122 \mathrm{msec}$.

\section{Accessibility}

Complete listings of the BASIC program, timing routines, card layout, and wiring schematics may be obtained by sending a self-addressed envelope and $\$ 2$ for postage to the first author.

\section{REFERENCES}

Brooks, J. O., III (1987). Enhancing and degrading visual stimuli. Behavior Research Methods, Instruments, \& Computers, 19, 260-269.

DORFMAN, D. (1987). Software timing of events in cognitive psychology experiments. Behavior Research Methods, Instruments, \& Computers, 19, 185-190.

Fraser, I. H., Lishman, J. R., \& Parker, D. M. (1987). Temporal manipulation of stimulus patterns using the Apple Il: Tachistoscopic and part presentation. Behavior Research Methods, Instruments, \& Computers, 19, 315-318.

Jensen, D. G. (1987). Facial perception studies using the Macintosh. Behavior Research Methods, Instruments, \& Computers, 19, 252-256.

LANCASTER, D. (1984). Enhancing your Apple II (Vol. 1, 2nd ed.). Indianapolis, IN: Sams.

LoFTus, G. R. (1982). Picture memory: Data and methodology. In C. R. Puff (Ed.), Handbook of research methods in human memory and cognition (pp. 258-287). New York: Academic Press.

Loftus, G. R., Gilluspie, S., Tigre, R. A., \& Nelson, W. W. (1984). An Apple II-based slide projector laboratory. Behavior Research Methods, Instruments, \& Computers, 16, 447-453.

PrILL, K. A. (1984). Picture stimuli for the Apple II computer. Behavior Research Methods, Instruments, \& Computers, 10, 327-328.

Snodgrass, J. G., Smith, B., Feenan, K., \& Corwin, J. (1987). Fragmenting pictures on the Apple Macintosh computer for experimental and clinical applications. Behavior Research Methods, Instruments, \& Computers, 19, 270-274. 
Appendix

Cost of System Components

\$Canadian \$U.S.*

Computer

Apple II + , 48K memory, 1 disk drive,

monochromatic monitor $\ldots \ldots \ldots \ldots \ldots \ldots \ldots \ldots \quad 3,195 \quad 2,332 \dagger$

Interfaces

Custom hardware card .

65

48

Lafayette 18010 voice-operated relay

with microphone and stand.

$479 \quad 350$

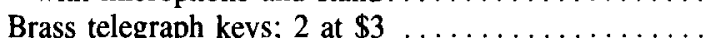

Assorted cables and connectors . . . . . . . . . .

6

$15-11$

6 outlet power bars; 2 at $\$ 25$

50

11
37

Optical Hardware

Kodak Ektagraphic carousel projectors;

Gerbrands G1166 shutters with

interface drivers; 3 at $\$ 718$

$2,154 \quad 1,573$

$1 \times 1 \mathrm{ft}$. Efston Science RE70,934B

rear-projection screen

20

Total

7,220

5,273

*U.S. prices are based on Canadian funds equivalent to $\$ 0.73$ U.S. †Currently Apple IIe computers with $128 \mathrm{~K}$ memory, 2 disk drives, and monitor retail for $\$ 2200$ Canadian, thus lowering total system costs to $\$ 6,175$ Canadian or $\$ 4,510$ U.S.

(Manuscript received July 24, 1987;

revision accepted for publication December 10, 1987.) 